\title{
Systemic infection of Staphylococcus aureus in postnatal woman: a unique finding
}

\author{
Annie Prasanthi ${ }^{1}$, Jiji Elizabeth Mathews ${ }^{1}$, Muruga Bharathy ${ }^{2}$, Swati Rathore ${ }^{1 *}$
}

\begin{abstract}
${ }^{1}$ Department of Obstetrics and Gynaecology, Christian Medical College, Vellore, Tamil Nadu, India
${ }^{2}$ Department of General Medicine Unit II, Christian Medical College, Vellore, Tamil Nadu, India
\end{abstract}

Received: 10 September 2020

Accepted: 28 October 2020

\author{
*Correspondence: \\ Dr. Swati Rathore, \\ E-mail: swatixrathore@gmail.com
}

Copyright: (c) the author(s), publisher and licensee Medip Academy. This is an open-access article distributed under the terms of the Creative Commons Attribution Non-Commercial License, which permits unrestricted non-commercial use, distribution, and reproduction in any medium, provided the original work is properly cited.

\begin{abstract}
Staphylococcus bacteraemia is the leading cause of hospital acquired and community acquired bacteraemia. Complications associated are difficult to recognise. Mortality is 20 to $40 \%$. This is a unique case discussing the metastatic spread of Staphylococcus infection following a wound infection. An informed consent was taken from the patient before publishing this case report.
\end{abstract}

Keywords: Staphylococcus aureus bacteraemia, Postnatal woman, Management

\section{INTRODUCTION}

Staphylococcus aureus is virulent and complicated bacteria. S. aureus blood stream infections are very common and difficult to treat. ${ }^{1}$ A variety of complications may arise and are difficult to identify early. Incidence of Staphylococcus aureus bacteremia is advancing in relation to newer treatments requiring invasive procedures like intravenous catheter, implants and increasing number of immunocompromised patients. ${ }^{2}$ Characteristic virulence of the pathogen in addition to transforming epidemiology of $S$. aureus bacteremia is demanding upgraded protocols and better antibiotics in management of $S$. aureus bacteremia and its complications. ${ }^{3}$

\section{CASE REPORT}

27-year-old Mrs. X, primigravida at 38 weeks gestation age with risk factors of mild anaemia and intrauterine growth restriction was induced. She delivered by outlet forceps for non-reassuring fetal status a baby girl weighing $1.9 \mathrm{~kg}$. On postnatal day 3 , she had high grade fever. She complained of pain in the episiotomy site and severe backache. On examination, episiotomy wound was unhealthy. Wound debridement was done under local anaesthesia. In spite of regular wound debridement and dressing she continued to have high grade fever. Hence blood culture and urine culture were sent and she was started on parenteral antibiotics empirically on postnatal day 5 .

\section{Investigations}

$\mathrm{TC}=7,600 /$ cumm, urine culture $>1$ lakh CFU/ML methicillin sensitive Staphylococcus aureus, blood culture=Methicillin sensitive Staphylococcus aureus.

Medicine consult was sought in view of Staphylococcus bacteraemia. X-ray spine followed by MRI spine with whole spine screening was advised. Multiple abscess were noted involving left psoas and iliacus muscles, right para renal soft tissue and inferior aspect of left sacroiliac joint and inflammatory soft tissue extends into the spinal canal causing thecal sac compression at L4-L5 vertebral levels (Figure 1). She was afebrile on day 5 of antibiotics. Antibiotics were continued till 6 weeks and alternate day blood cultures were sent and by 10 days the blood culture showed no growth. Transthoracic and transoesophageal ECHO was done to rule out infective endocarditis. ECHO was normal. She was stable and was discharged on $25^{\text {th }}$ postnatal day. 

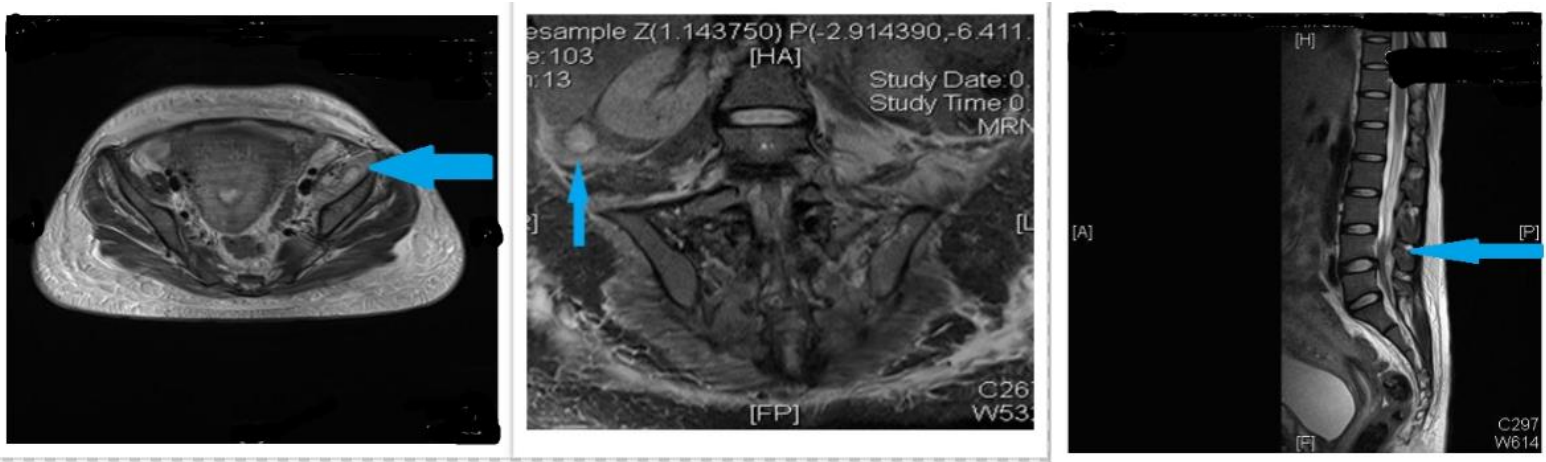

Figure 1: MRI image showing multiple abscess involving left psoas and iliacus muscles, right para renal soft tissue and inferior aspect of left sacroiliac joint and inflammatory soft tissue extends into the spinal canal causing thecal sac compression at L4-L5 vertebral levels.

\section{DISCUSSION}

Staphylococcus aureus is a gram-positive bacterium and is a part of normal flora of the body. S. aureus has an ability to up-regulate virulence factors under stressful stimuli. It invades the bloodstream and evade host immunological responses. They form protective biofilms. This develops resistance to several antibiotics. ${ }^{2}$ The clinical approach to $S$. aureus bacteraemia consists of careful history and physical examination, infectious disease consultation, and diagnostic evaluation including ECHO and additional imaging as needed. ${ }^{4}$ Failure to identify complications of $S$. aureus bacteraemia may lead to catastrophic complications (e.g., death, paralysis) or relapsing bacteraemia due to inadequate treatment. Early and aggressive antibiotic therapy and removal of source of infection are important for optimal management. Penicillin is the drug of choice for $S$. aureus. Cefazoline is an effective alternative. Patients with bacteraemia with a removable focus of infection may be treated within 2 weeks of intravenous therapy from the first negative blood culture. ${ }^{5}$

\section{Significance}

This patient was successfully managed by promptly diagnosing the episiotomy wound infection with dissemination. All the possible metastatic sites were checked. She was started on appropriate antibiotics and blood culture was negative by $10^{\text {th }}$ day of antibiotics. She received antibiotics for 6 weeks and she was followed up regularly. Metastatic infection was successfully treated and documented by repeat imaging.

\section{CONCLUSION}

Early detection of this clinical condition is essential to prevent morbidity and mortality care provider should be careful with postnatal mothers.

Funding: No funding sources

Conflict of interest: None declared

Ethical approval: Not required

\section{REFERENCES}

1. Wisplinghoff $H$, Bischoff T, Tallent SM, Seifert H, Wenzel RP, Edmond MB. Nosocomial bloodstream infections in US hospitals: analysis of 24,179 cases from a prospective nationwide surveillance study. Clin Infect Dis. 2004;39:309-17.

2. Christoph K. Nabera. Staphylococcus aureus Bacteremia: Epidemiology, Pathophysiology, and Management Strategies. Clin Infect Dis. 2009;48:231-7.

3. Lodise TP, McKinnon PS, Swiderski L, Rybak MJ. Outcomes analysis of delayed antibiotic treatment for hospital-acquired Staphylococcus aureus bacteremia. Clin Infect Dis. 2003;36:1418-23.

4. Holland TL, Arnold C, Fowler VG Jr. Clinical management of Staphylococcus aureus bacteremia: a review. JAMA. 2014;312:1330.

5. Miller LG. Clinical and epidemiologic characteristics cannot distinguish community-associated methicillin-resistant Staphylococcus aureus infection from methicillin-susceptible $S$. aureus infection: a prospective investigation. J Infect Dis. 2007;44(4):471-82.

Cite this article as: Prasanthi A, Mathews JE, Bharathy M, Rathore S. Systemic infection of Staphylococcus aureus in postnatal woman: a unique finding. Int J Reprod Contracept Obstet Gynecol 2021;10:1183-4. 